\title{
Non-linear losses study in strongly coupled piezoelectric device for broadband energy harvesting
}

\author{
David GIBUS ${ }^{1,2}{ }^{*}$, Pierre GASNIER ${ }^{2}$, Adrien MOREL ${ }^{2}$, Nicolas Garraud ${ }^{2}$ and Adrien BADEL ${ }^{1}$ \\ ${ }^{1}$ SYMME, Université Savoie Mont-Blanc, F-74000, Annecy, France \\ 2 Université Grenoble Alpes, CEA, LETI, MINATEC, F-38000 Grenoble, France \\ *david.gibus@univ-smb.fr
}

\begin{abstract}
Piezoelectric vibration harvesters with strong electromechanical coupling coefficients have recently been combined with nonlinear electrical techniques capable of tuning their resonant frequency as a solution to provide energy to wireless sensor nodes from wideband vibrations. To be fully competitive, this approach requires piezoelectric generators with strong global electromechanical coupling coefficients $\mathrm{k}^{2}$. However, the presence of non-linear dielectric and piezoelectric losses in piezoelectric materials significantly reduces the power harvested when strongly coupled materials are used. This paper presents a non-linear model that allows for a better consideration of losses for such piezoelectric harvesters. An experimental validation is performed with a strongly coupled cantilever based on a PMN-PT material $\left(\mathrm{k}^{2}=16 \%\right)$. The results reveal the importance of considering non-linear dielectric losses. Indeed, we show thanks to the model that neglecting these losses can induce an $18 \%$ overestimation of the harvested power for the presented prototype driven at $0.5 \mathrm{~m} / \mathrm{s}^{2}$ amplitude ambient acceleration.
\end{abstract}

\begin{tabular}{|c|c|c|c|}
\hline \multicolumn{4}{|c|}{ Nomenclature } \\
\hline$M_{t}$ & Mass of the proof mass & $\omega$ & Vibration pulsation \\
\hline$I_{t}$ & $\begin{array}{l}\text { Rotary inertia of the proof mass according to } \\
\text { its center of gravity }\end{array}$ & $f_{\text {ext }}$ & Vibration frequency \\
\hline$D_{t}$ & $\begin{array}{l}\text { Distance between the free end of the beam and } \\
\text { the center of gravity of the proof mass }\end{array}$ & $\phi(x)$ & Vibration mode shape \\
\hline$J_{t}$ & $\begin{array}{l}\text { Ratio between the rotation inertia and the } \\
\text { mass }\end{array}$ & $k_{31}^{2}$ & $\begin{array}{l}\text { Material coupling coefficient of the } \\
\text { piezoelectric material }\end{array}$ \\
\hline$L_{b}$ & Beam length & $k^{2}$ & $\begin{array}{l}\text { Global electromechanical coupling coefficient } \\
\text { of the piezoelectric harvester }\end{array}$ \\
\hline$L_{m}$ & Proof mass length & $K$ & Equivalent linear stiffness \\
\hline$H_{m}$ & Proof mass height & $B_{f}$ & Forcing term \\
\hline$B$ & Beam and mass width & $M$ & Equivalent mass \\
\hline $\mathcal{V}_{p}$ & Substrate volume & $\Theta$ & Linear coupling term \\
\hline $\mathcal{V}_{p}$ & Volume of piezoelectric material & $C_{p}$ & Equivalent clamped capacitance \\
\hline$m$ & Mass per unit length of the bimorph & $\alpha$ & Linear coupling term per unit length \\
\hline$h_{p}$ & Piezoelectric patches thickness & $\widetilde{\theta_{2}}$ & Second order coupling term per unit length \\
\hline$h_{s}$ & Substrate thickness & $\widetilde{k_{2}}$ & Second order bending stiffness per unit length \\
\hline$e_{31}^{e f}$ & Linear piezoelectric equivalent coefficient & $K_{2}$ & Nonlinear stiffness \\
\hline
\end{tabular}




\begin{tabular}{|c|c|c|c|}
\hline$d_{31}$ & $\begin{array}{l}\text { Coefficient of the piezoelectric constant } \\
\text { matrix }\end{array}$ & $\Theta_{2}$ & Nonlinear coupling term \\
\hline$s_{11}^{E}$ & $\begin{array}{l}\text { Coefficient of the compliance matrix of the } \\
\text { piezoelectric material }\end{array}$ & $R_{\text {load }}$ & Resistive load \\
\hline$\epsilon_{33}^{T}$ & $\begin{array}{l}\text { Coefficient of the free dielectric matrix of the } \\
\text { piezoelectric material }\end{array}$ & $b_{1}$ & Linear mechanical structural loss coefficient \\
\hline$\epsilon_{33}^{e f}$ & $\begin{array}{l}\text { Linear dielectric permittivity equivalent } \\
\text { coefficient }\end{array}$ & $b_{2}$ & Second order structural loss coefficient \\
\hline$c_{11}^{e f}$ & Linear elastic equivalent coefficient & $d_{1}$ & Linear dielectric loss coefficient \\
\hline$c_{111}$ & Nonlinear elastic constant & $d_{2}$ & second order dielectric loss coefficient \\
\hline$e_{311}$ & Nonlinear piezoelectric equivalent constant & $a, b$ & $\begin{array}{l}\text { Coefficients that define the beam mode shape } \\
\text { expression }\end{array}$ \\
\hline$Y_{S}$ & Young modulus of the substrate & $w_{L_{b}}$ & Beam end relative velocity displacement \\
\hline$Y I$ & Linear bending stiffness & $w_{B}$ & Base displacement of the beam \\
\hline$x$ & Longitudinal spatial coordinate & $\mathcal{A}_{B}$ & Acceleration amplitude of the beam base \\
\hline$y$ & Lateral spatial coordinate & $w(x)$ & Transverse deflection of the beam \\
\hline$z$ & Transversal spatial coordinate & $H$ & $\begin{array}{l}\text { Nonlinear electric enthalpy density of the } \\
\text { piezoelectric material }\end{array}$ \\
\hline$S_{1}$ & Longitudinal strain & $L$ & Lagrangian \\
\hline$E_{3}$ & Transverse electric field & $W_{\text {ext }}$ & External work \\
\hline$v$ & Voltage accross the piezoelectric electrodes & $T$ & Total kinetic energy \\
\hline$V$ & Voltage amplitude & $F_{n}$ & Non-conservative force \\
\hline$r$ & Generalized spatial coordinate & $I_{n}$ & Leakage current \\
\hline$R$ & $\begin{array}{l}\text { Amplitude of the generalized sp } \\
\text { coordinate } r\end{array}$ & $\dot{\dot{a}}$ & Temporal derivative \\
\hline$\lambda$ & Flux linkage & $\square^{\prime}$ & Spatial derivative according to $x$ coordinate \\
\hline
\end{tabular}

\section{Introduction}

Vibration energy harvesting is recognized as a relevant solution to supply electrical power to wireless sensors nodes [1-3]. In order to reach sufficient power density, the use of electromechanical resonators have been shown required for inertial energy harvesting $[4,5]$. However, the resulting narrow frequency bandwidth of vibration energy harvesters is still an important issue [6,7]. A promising solution to address this problem is to use nonlinear electrical techniques $[8,9]$ and power management circuits $[10,11]$ able to tune the resonant frequency of piezoelectric harvesters. For example, this solution made it possible to expect a relative bandwidth of $43 \%$ with a PZN-PT-based cantilever [9]. In order for this approach to be effective, it is necessary to employ piezoelectric harvesters with very strong global electromechanical coupling coefficients $k^{2}\left(k^{2}>10 \%\right)$. Proposals for the design of such harvesters have therefore recently been made in the literature $[12,13]$ and offer promising perspectives for broadband vibration energy harvesting. Nevertheless, the presence of nonlinear dielectric losses in the piezoelectric materials have been shown to considerably reduce the harvested power when strongly coupled materials are used [14]. Such dielectric losses must be taken into account in the models as neglecting them can lead to a significant error in the expected harvested power. Since a model to predict the behavior of the harvester taking into account non-linear dielectric losses is absent from the state of the art, this paper proposes a new nonlinear model corroborated by experimental results for strongly coupled energy harvester.

The linear losses of piezoelectric materials have already been studied in the prior art for various piezoelectric devices $[15,16]$. As an example, Uchino et al. expressed the necessity to consider a piezoelectric loss tangent 
coefficient especially for actuator applications [17]. Recently, Wild et al. discussed the difficulty to distinguish dissipation origin (mechanical, dielectric and piezoelectric) depending on the piezoelectric constitutive equation form [18]. Concerning energy harvesting applications, dielectric and piezoelectric losses in piezoelectric materials are often neglected $[19,20]$ as they don't have a significant influence for low and moderately coupled devices. The electric field experienced is indeed usually weak in the materials. Nevertheless, the consideration of a dielectric loss coefficient has been shown necessary for devices that include strongly coupled materials [14,21]. While models to consider linear dielectric losses have been proposed in the literature [21], nonlinear dielectric losses can be experienced in the very strongly coupled devices. Indeed, Morel et al. demonstrated the increase of the equivalent dielectric loss tangent on a PZN-PT based cantilever under an increasing electric field [14]. They also observed such nonlinear dielectric losses with the increase of the vibration amplitude. They indeed showed an experimental decrease of $30 \%$ of the power due to the increasing electric field in the material. Therefore, considering nonlinear dielectric losses is necessary to model the behaviour of vibration energy harvesters in the case of prototypes involving strongly coupled materials.

In this paper, we introduce a model that takes material nonlinearities of strongly coupled piezoelectric harvesters into account. The model, based on the Rayleigh method, is introduced in section 2. In order to validate this model, a strongly coupled prototype and the experiments conducted are then presented in section 3 . The section 4 is dedicated to results presentation and discussion. In this last section, we discuss the importance of considering a dielectric nonlinear losses coefficient.

\section{Modelling}

\subsection{Discussion about dielectric loss coefficients}

This section deals with the loss considerations made in the present work. Losses in piezoelectric materials have been discussed in various ways in the literature $[17,18]$. It seems important to clearly state the intention of this paper and the definition of the loss coefficients used.

As mentioned by Wild et al. [18], the terms "dielectric loss", "mechanical loss" and "piezoelectric loss" are intended to describe physical contributions that differ depending on the considered form of the piezoelectric constitutive equation (the $e$-form, $d$-form, $h$-form and $g$-form [22]). For the analysis of vibration energy harvesters, the base acceleration is usually fixed and the output voltage on the electrodes is measured, and therefore, the $e$ form is used. For this form of constitutive equation, the energy loss per cycle that increases with the displacement (strain in case of local consideration) at constant voltage (electrical field in case local consideration) is considered in the term of "mechanical loss". Moreover, the term of "dielectric loss" represents the energy loss per cycle that increases with the output voltage at constant displacement. Finally, the "piezoelectric" term induces an effect that increases with both displacement and electric field. This effect can induce either losses or an increase in the harvested power [23]. The above definitions of "mechanical", "piezoelectric" and "dielectric" losses will be used in this paper.

According to the work of Leadenham and Erturk [19], a second-order nonlinear mechanical loss coefficient must be considered in order to model the behaviour of PZT based cantilevers. Both linear and non-linear mechanical dissipations must be taken into account in the model. The energy loss per cycle due to linear mechanical loss $U_{m}^{\mathrm{lin}}$ is proportional to the square of the relative displacement of the beam $R$ as expressed in (1) and the energy loss per cycle due to nonlinear mechanical loss $U_{m}^{\text {nonlin }}$ is proportional to the cube of the relative displacement of the beam $R$ as mentioned in (2).

$$
\begin{gathered}
U_{m}^{\operatorname{lin}} \propto R^{2} \\
U_{m}^{\text {nonlin }} \propto R^{3}
\end{gathered}
$$

The model proposed in [19] did not consider piezoelectric and dielectric losses as this did not seem necessary for the studied PZT-based cantilevers. Considering linear dielectric losses, the energy loss per cycle $U_{d}^{\text {lin }}$ is proportional to the output voltage amplitude $V$ of the harvested as expressed in (3) where $V$ is the voltage amplitude.

$$
U_{d}^{\operatorname{lin}} \propto V^{2}
$$


Considering a second-order nonlinear dielectric loss coefficient, the energy loss per cycle due to electrical nonlinear loss $U_{d}^{\text {nonlin }}$ is proportional to the cube of the voltage amplitude.

$$
U_{d}^{\text {nonlin }} \propto V^{3}
$$

Since the experimentally observed dissipations as a function of the voltage are due to both piezoelectric and dielectric hysteresis [24], piezoelectric loss coefficients should be considered in addition to dielectric loss coefficients to finely model the harvesters behavior. However, we show in this paper that only dielectric loss coefficients are sufficient for the phenomenological modelling of the performance of vibration energy harvesters. Dielectric losses and piezoelectric losses have equivalent influences on the mean power harvested by piezoelectric vibration energy harvesters, as long as the vibration frequency remains in the vicinity of the resonant frequency of the harvester. Since off-resonance states are, in most cases, out of interest in energy harvesting applications (because of the low harvested power level), distinguishing these two kind of losses appears unnecessary

Therefore, we propose a nonlinear model that takes into account linear and nonlinear dielectric losses in addition to linear and nonlinear mechanical losses as proposed by Leadenham and Erturk [19]. The model also considers a nonlinear stiffness coefficient and a nonlinear coupling coefficient in order to take the mechanical softening of the piezoelectric material into account. The device studied is presented in the following section. The model is then introduced in section 2.3.

\subsection{Design and parameters of the system}

The studied harvester consists of a bimorph cantilever with a bulky proof mass at the tip end (Figure 1.a) and for which the two piezoelectric layers have the same length $\left(L_{b}\right)$ and the same width $(B)$ as the substrate. The piezoelectric layers have the same thicknesses $\left(h_{p}\right)$ and are completely covered with electrodes on the bottom and top surfaces, which are connected in parallel (Figure 2). Figure 1.b shows the problem parameters of the proposed cantilever while bending. The proof mass, symmetrical with respect to the neutral axis of the beam, is modelled with an equivalent point mass defined by a mass $M_{t}$ and a rotary inertia $I_{t}$ placed at a distance $D_{t}$ from the free end of the beam on the neutral axis.

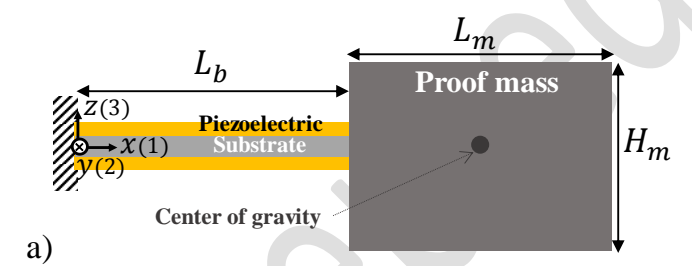

b)

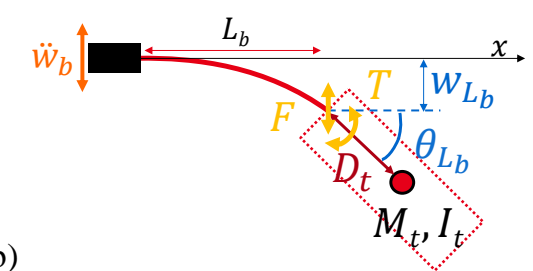

Figure 1: a) Cantilever with proof mass and b) cantilever during bending.

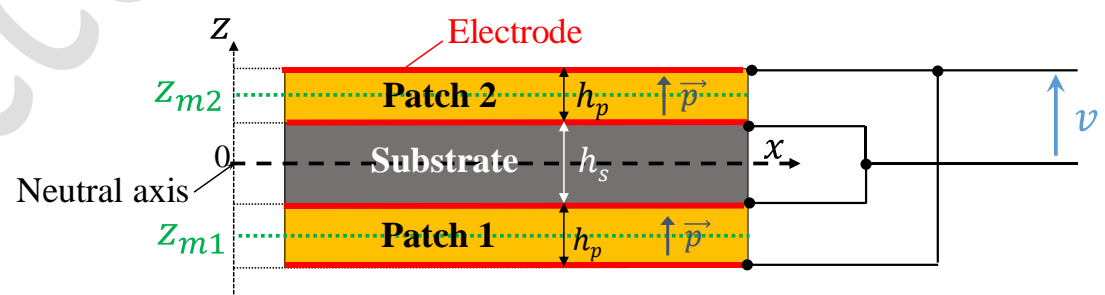

Figure 2: Beam layers and electrode connection. $v$ represents the voltage across the electrodes.

As the beam length is much larger compared to its thickness $\left(L_{b}>10\left(2 h_{p}+h_{s}\right)\right)$, the Euler-Bernoulli theory can be used. Furthermore, as the beam length is much larger than the width $\left(L_{b}>5 B\right)$, the plane stress assumption is considered. Finally, due to the electrodes' architecture, the displacement field and the electric field are only considered in the transverse direction ( $E_{3}$ and $D_{3}$ along the $z$ axis).

The model introduced here is derived from the two degree of freedom linear model that we presented in [12], focusing on the first resonant mode. The Hamilton principle and the Rayleigh method are used to determine the equation of motion and the equation of current. Unlike the model in [12], nonlinear terms are introduced in the enthalpy expression to consider the mechanical softening and piezoelectric coefficient nonlinearity. Moreover, 
linear and nonlinear losses are considered as an external work by adding non-conservative forces and leakage currents.

The Rayleigh method is detailed, and the equations of motion and current are determined in section 2.3. The expression of the first mode shape is determined in section 2.4 and the equation is solved to find the steady state solution in the case of sinusoidal excitation in section 2.5 .

\subsection{Hamilton principle}

The Hamilton principle applied to the piezoelectric harvester is described in (5) where $\delta$ represents virtual variations of the quantities between time $t_{0}$ and $t_{1}$. $L$ is the Lagrangian that represents the difference of the kinetic energy $T$ and the total potential energy $U$ as expressed in (6). $W_{\text {ext }}$ is the external work that considers the harvested energy and the losses.

$$
\begin{gathered}
\int_{t_{0}}^{t_{1}}\left(\delta L+\delta W_{\text {ext }}\right) d t=0 \\
L=T-U
\end{gathered}
$$

The total potential energy $U$ of the piezoelectric bimorph is the sum of the potential energies of the substrate, $U_{s}$, and of the piezoelectric material $U_{p}$ as expressed in (7). The expressions of $U_{s}$ and $U_{p}$ are given in (8) and (9) respectively. The equivalent stiffness of the substrate $Y_{s}^{e f}$ is equal the Young modulus $Y_{s}$ of the substrate as the plane stress assumption is considered. $S_{1}$ is the longitudinal strain in the material. $H$ represents the enthalpy of the piezoelectric material. $\mathcal{V}_{p}$ and $\mathcal{V}_{s}$ represent the volumes of the piezoelectric patches and the substrate respectively.

$$
\begin{gathered}
U=U_{p}+U_{s} \\
U_{s}=\frac{1}{2} \int_{\mathcal{V}_{s}} Y_{s}^{e f} S_{1}^{2} d \mathcal{V}_{s} \\
U_{p}=\int_{\mathcal{V}_{p}} H d V_{p}
\end{gathered}
$$

According to the work of Leadenham and Erturk [19], the nonlinear electric enthalpy density expression in the piezoelectric material is given by equation (10) where $E_{3}$ is the electric field, $c_{11}^{e f}$ and $e_{31}^{e f}$ represent the linear elastic and piezoelectric equivalent coefficients respectively. $\epsilon_{33}^{e f}$ is the linear dielectric permittivity equivalent coefficient. $c_{111}$ is the nonlinear elastic constant and $e_{311}$ is the nonlinear piezoelectric constant. The function sgn() represents the sign function as expressed in (11). As the plane stress assumption is considered, the equivalent linear parameters are given from the matrix coefficients in equations (12) to (14).

$$
\begin{gathered}
H=\frac{1}{2} c_{11}^{e f} S_{1}^{2}+\frac{1}{3} c_{111} S_{1}^{3} \operatorname{sgn}\left(S_{1}\right)-e_{31}^{e f} S_{1} E_{3}-\frac{1}{2} e_{311} S_{1}^{2} \operatorname{sgn}\left(S_{1}\right) E_{3}-\frac{1}{2} \epsilon_{33}^{e f} E_{3}^{2} \\
\operatorname{sgn}(f)= \begin{cases}1 & \text { if } f \geq 0 \\
-1 & \text { if } f<0\end{cases} \\
\epsilon_{33}^{e f}=\epsilon_{33}^{T}-\frac{d_{31}^{2}}{s_{11}^{E}} \quad \text { (12) } \quad c_{11}^{e f}=\frac{1}{s_{11}^{E}}
\end{gathered}
$$

The longitudinal strain in the beam is given in (15) according to the Euler-Bernoulli theory where $w(x, t)$ is the transverse (in the $z$ direction) deflection of the beam. The apostrophes represent the spatial derivative according to $x$ coordinate. As discussed in [25], the electric field must be considered variable across the patches thicknesses for strongly coupled piezoelectric materials. Then the expression of the transverse electric field is given by (16) where $\lambda$ is the flux leakage as introduced in [26] and expressed in (17). $z_{m}=\left(h_{p}+h_{s}\right) / 2$ is the center position of the upper piezoelectric patch. With this definition $-z_{m}$ is the center position of the lower piezoelectric patch.

$$
S_{1}=-z w^{\prime \prime}(x, t)
$$




$$
\begin{gathered}
E_{3}=-\frac{\dot{\lambda}}{h_{p}} \operatorname{sgn}(z)+\frac{e_{31}^{e f}}{\epsilon_{33}^{e f}}\left(z-z_{m} \operatorname{sgn}(z)\right) w^{\prime \prime}(x, t) \\
\dot{\lambda}=v
\end{gathered}
$$

The total potential energy $U$ can finally be obtained using the equations (8) to (10) and (15) and (16) and is expressed in (18) where $Y I$ is the linear bending stiffness expressed in (19). $\widetilde{k_{2}}$ is the second order mechanical bending stiffness per unit length expressed in (20), $\alpha$ is the linear coupling term per unit length expressed in (21), $\widetilde{\theta_{2}}$ is the second order coupling term per unit length expressed in (22), $C_{p}$ is the equivalent clamped capacitance expressed in (23).

$$
\begin{gathered}
U=\frac{1}{2} \int_{0}^{L_{b}}\left\{Y I\left(w^{\prime \prime}\right)^{2}+\frac{1}{3} \widetilde{k_{2}}\left(w^{\prime \prime}\right)^{3} \operatorname{sgn}\left(w^{\prime \prime}\right)-\left[\alpha w^{\prime \prime}+\widetilde{\theta_{2}}\left(w^{\prime \prime}\right)^{2} \operatorname{sgn}\left(w^{\prime \prime}\right)\right] \dot{\lambda}\right\} d x-\frac{1}{2} C_{p} \dot{\lambda}^{2} \\
Y I=B\left[Y_{s}^{e f} \frac{h_{s}^{3}}{12}+c_{11}^{e f}\left(\frac{2}{3}\left(h_{p}+\frac{h_{s}}{2}\right)^{3}-\frac{h_{s}^{3}}{12}+k_{e_{31}}^{2} \frac{h_{p}^{3}}{6}\right)\right] \\
\widetilde{k_{2}}=B \frac{1}{2} h_{p}\left[c_{111}\left(h_{p}^{3}+4 h_{p}^{2} h_{s}+3 h_{p} h_{s}^{2}+h_{s}^{3}\right)+\frac{e_{31}^{e f}}{\epsilon_{33}^{e f}} e_{311}\left(h_{p}^{3}+h_{p}^{2} h_{s}\right)\right] \\
\alpha=e_{31}^{e f} B\left(h_{p}+h_{s}\right) \\
\widetilde{\theta_{2}}=\frac{1}{6} e_{311} B\left(4 h_{p}^{2}+6 h_{p} h_{s}+3 h_{s}^{2}\right) \\
C_{p}=\frac{B 2 L_{b} \epsilon_{33}^{e f}}{h_{p}}
\end{gathered}
$$

The total kinetic energy $T$ considered in the Hamilton principle consists of the kinetic energies of the beam and of the proof mass including its rotary inertia is expressed in (24). $m$ represents the mass per unit length of the bimorph expressed in (25) where $\rho_{s}$ and $\rho_{p}$ are the densities of the piezoelectric material and of the substrate respectively. $\dot{w}_{B}$ is the base (clamped end) velocity, $w_{L b}$ is the beam end relative velocity, ${\dot{w_{L b}}}^{\prime}$ is the beam end speed of rotation.

$$
\begin{gathered}
T=\frac{1}{2} \int_{0}^{L_{b}} m\left[\dot{w}+\dot{w}_{B}(t)\right]^{2} d x_{1} \\
+\left[M_{t}\left(\dot{w_{L_{b}}}+\dot{w}_{B}\right)^{2}+M_{t} D_{t}\left(\left(\dot{w}_{L_{b}}+\dot{w}_{B}\right){\dot{w_{L_{b}}}}{ }^{\prime}+{\dot{w_{L_{b}}}}^{\prime}\left(\dot{w}_{L_{b}}+\dot{w}_{B}\right)\right)\right. \\
\left.+\left(M_{t} D_{t}^{2}+I_{t}\right){\dot{w_{L_{b}}}}{ }^{2}\right] \\
m=B\left(h_{s} \rho_{s}+2 h_{p} \rho_{p}\right)
\end{gathered}
$$

Using the Rayleigh approach, $w(x, t)$ can be expressed by the mode shape $\phi(x)$ multiplied by a generalized mechanical coordinate $r(t)$ as given in (26). $\phi(x)$ is the first resonant frequency mode shape and $r(t)$ describes the displacement of a point on the beam as a function of time. Consequently, we can define two generalized coordinates that are the displacement at the end of the beam $r(t)$ and the flux leakage $\lambda(t)$. The variation of the Lagrangian can then be expressed as (27).

$$
\begin{gathered}
w(x, t)=\phi(x) r(t) \\
\delta L(r, \dot{r}, \dot{\lambda})=\frac{\partial L}{\partial \dot{r}} \delta \dot{r}+\frac{\partial L}{\partial r} \delta r+\frac{\partial L}{\partial \dot{\lambda}} \delta \dot{\lambda}
\end{gathered}
$$

As discussed in section 2.2, the mechanical losses and dielectric losses are considered by a non-conservative force $F_{n}$ and a leakage current $I_{n}$ that are expressed in (28) and (29) respectively, where $b_{1}$ is the linear mechanical 
structural loss coefficient, $b_{2}$ is a second order structural loss coefficient, $d_{1}$ is a linear dielectric loss coefficient, $d_{2}$ is a second order dielectric loss coefficient. Considering that the harvester is connected to a resistive load $R_{\text {load }}$, the variation of the external work is expressed by equation (30).

$$
\begin{gathered}
F_{n}=-\left[b_{1} r \operatorname{sgn}(r)+b_{2} r^{2}\right] \operatorname{sgn}(\dot{r}) \\
I_{n}=-\left[d_{1} \dot{v} \operatorname{sgn}(v)+d_{2} v \dot{v}\right] \operatorname{sgn}(\dot{v}) \\
\delta W_{\text {ext }}=-\frac{\dot{\lambda}}{R_{\text {load }}} \delta \lambda-\left[b_{1} r \operatorname{sgn}(r)+b_{2} r^{2}\right] \operatorname{sgn}(\dot{r}) \delta r-\left[d_{1} \ddot{\lambda} \operatorname{sgn}(\dot{\lambda})+d_{2} \dot{\lambda} \ddot{\lambda}\right] \operatorname{sgn}(\ddot{\lambda}) \delta \lambda
\end{gathered}
$$

Integrating by parts the Lagrangian variation in (27) and using the work expression in (30), the Hamilton principle given in (5) becomes the equation (31).

$$
\begin{aligned}
\int_{t_{0}}^{t_{1}}\left\{\left[-\frac{\partial}{\partial t}\left(\frac{\partial L}{\partial r}\right)\right.\right. & \left.+\frac{\partial L}{\partial r}-\left[b_{1} r \operatorname{sgn}(r)+b_{2} r^{2}\right] \operatorname{sgn}(\dot{r})\right] \delta r \\
& \left.+\left[-\frac{\partial}{\partial t} \frac{\partial L}{\partial \dot{\lambda}}-\frac{\dot{\lambda}}{R}-\left[d_{1} \ddot{\lambda} \operatorname{sgn}(\dot{\lambda})+d_{2} \dot{\lambda} \ddot{\lambda}\right] \operatorname{sgn}(\ddot{\lambda})\right] \delta \lambda\right\} d t=0
\end{aligned}
$$

Replacing the Lagrangian with the expressions of the potential energy (18) and of the kinetic energy (24) in equation (31) allows to deduce equations (32) and (33), where the equivalent mass $M$, the linear stiffness $K$, the linear coupling term $\Theta$, the forcing term $B_{f}$, the nonlinear stiffness $K_{2}$ and the nonlinear coupling term $\Theta_{2}$ are expressed in equations (34) to (39).

$$
\begin{gathered}
M \ddot{r}+K r+K_{2} r^{2} \operatorname{sgn}(r)-\Theta v-\Theta_{2} v r \operatorname{sgn}(r)+\left(b_{1} r \operatorname{sgn}(r)+b_{2} r^{2}\right) \operatorname{sgn}(\dot{r})=-B_{f} \ddot{w}_{B} \\
\Theta \dot{r}+\Theta_{2} r \dot{r} \operatorname{sgn}(r)+C_{p} \dot{v}+\frac{v}{R_{\text {load }}}+\left(d_{1} \dot{v} \operatorname{sgn}(v)+d_{2} v \dot{v}\right) \operatorname{sgn}(\dot{v})=0 \\
M=m \int_{0}^{L_{b}} \phi(x)^{2} d x+M_{t}\left[\phi\left(L_{b}\right)\right]^{2}+2 M_{t} D_{t} \phi^{\prime}\left(L_{b}\right) \phi\left(L_{b}\right)+\left(I_{t}+M_{t} D_{t}^{2}\right)\left[\phi^{\prime}\left(L_{b}\right)\right]^{2} \\
K=Y I \int_{0}^{L_{b}}\left(\phi^{\prime \prime}(x)\right)^{2} d x \\
\Theta=\alpha \phi^{\prime}\left(L_{b}\right) \\
B_{f}=m \int_{0}^{L_{b}} \phi(x) d x+M_{t} \phi\left(L_{b}\right)+D_{t} M_{t} \phi^{\prime}\left(L_{b}\right) \\
K_{2}=\widetilde{k_{2}} \int_{0}^{L_{b}} \operatorname{sgn}\left(\phi^{\prime \prime}(x)\right)\left(\phi^{\prime \prime}(x)\right)^{3} d x \\
\Theta_{2}=\widetilde{\theta_{2}} \int_{0}^{L_{b}} \operatorname{sgn}\left(\phi^{\prime \prime}(x)\right)\left(\phi^{\prime \prime}(x)\right)^{2} d x
\end{gathered}
$$

\subsection{Beam mode shape expression}

The mode shape $\phi(x)$ determination of the first bending resonant mode of a cantilever with proof mass was previously detailed in [12]. As the beam mass is considered small compared to the mass of the proof mass, the mode shape can be expressed analytically as a function of the proof mass parameters and the beam length. If the expression of the mode shape is normalized by the beam end displacement $\left(\phi\left(L_{b}\right)=1\right), r(t)$ represents the beam displacement at $x=L_{b}$ and $\phi(x)$ is expressed by (40). $a$ and $b$ are coefficients that are expressed in the (41) and (42) respectively. $\beta$ is the rotation amplitude to deflection amplitude ratio as introduced in [12] and expressed in (43), where $J_{t}$ is the ratio between the rotation inertia and the mass defined as $J_{t}=I_{t} / M_{t}$.

$$
\phi(x)=\frac{x^{2}\left(3 b-a \frac{x}{L_{b}}+3 a\right)}{6}
$$




$$
\begin{aligned}
& \quad a=\frac{6\left(2-L_{b} \beta\right)}{L_{b}^{2}} \quad(41) \quad b=\frac{2\left(2 L_{b} \beta-3\right)}{L_{b}{ }^{2}} \\
& \beta=-\frac{3\left(2 D_{t}+L_{b}\right)}{3 J_{t}-\left(9 D_{t}^{4}+18 D_{t}^{3} L_{b}+15 D_{t}^{2} L_{b}^{2}+18 D_{t}^{2} J_{t}+6 D_{t} L_{b}^{3}+18 D_{t} L_{b} J_{t}+L_{b}^{4}+3 L_{b}^{2} J_{t}+9 J_{t}^{2}\right)^{\frac{1}{2}}+3 D_{t}^{2}-L_{b}^{2}}
\end{aligned}
$$

\subsection{Model resolution}

As done in [19], the method of harmonic balance is used to solve the nonlinear differential equations (32) and (33). For an harmonic base acceleration with constant amplitude $\left(\ddot{w}_{B}(t)=\mathcal{A}_{B} \cos (\Omega t)\right.$ ), we assume that the displacement $x$ and the voltage $v$ are harmonic and we consider the first harmonic terms of the Fourier series $R_{1}$, $R_{2}, V_{1}$ and $V_{2}$ as expressed in (44) and (45).

$$
\begin{aligned}
& r(t)=R_{1} \cos (\omega t)+R_{2} \sin (\omega t) \\
& v(t)=V_{1} \cos (\omega t)+V_{2} \sin (\omega t)
\end{aligned}
$$

Replacing $r(t)$ and $v(t)$ in equations (32) and (33) and considering the first harmonic terms in the application of the harmonic balance results to equations (46) to (49) where $R$ and $V$ are the displacement and voltage amplitudes given by $\sqrt{R_{1}^{2}+R_{2}^{2}}$ and $\sqrt{V_{1}^{2}+V_{2}^{2}}$ respectively.

$$
\begin{gathered}
-M \omega^{2} R_{1}+\left(\frac{2}{\pi} b_{1}+\frac{4}{3 \pi} b_{2} R\right) R_{2}+\left(K+\frac{8}{3 \pi} K_{2} R\right) R_{1}-\Theta V_{1}-\frac{4}{3 \pi} \Theta_{2}\left[\frac{\left(2 R_{1}^{2}+R_{2}^{2}\right) V_{1}+R_{1} R_{2} V_{2}}{R}\right] \\
+B_{f} \mathcal{A}_{B}=0 \\
-M \omega^{2} R_{2}-\left(\frac{2}{\pi} b_{1}+\frac{4}{3 \pi} b_{2} R\right) R_{1}+\left(K+\frac{8}{3 \pi} K_{2} R\right) R_{2}-\Theta V_{2}-\frac{4}{3 \pi} \Theta_{2}\left[\frac{\left(2 R_{2}^{2}+R_{1}^{2}\right) V_{2}+R_{1} R_{2} V_{1}}{R}\right]=0 \\
C_{p} \omega V_{2}+\frac{1}{R_{\text {load }}} V_{1}+\left(\Theta+\frac{4}{3 \pi} \Theta_{2} R\right) \omega R_{2}+\frac{2}{\pi} d_{1} \omega V_{1}+d_{2} \frac{4}{3 \pi} V \omega V_{1}=0 \\
C_{p} \omega V_{1}-\frac{1}{R_{\text {load }}} V_{2}+\left(\Theta+\frac{4}{3 \pi} \Theta_{2} R\right) \omega R_{1}-\frac{2}{\pi} d_{1} \omega V_{2}-d_{2} \frac{4}{3 \pi} V \omega V_{2}=0
\end{gathered}
$$

Equations (46) to (49) are then solved thanks to Newton-Raphson algorithm in order to determine the voltage amplitudes $\left(V_{1}\right.$ and $\left.V_{2}\right)$ and relative displacement amplitudes $\left(R_{1}\right.$ and $\left.R_{2}\right)$ at given excitation pulsation $\omega$, resistive load $R_{\text {load }}$ and base acceleration amplitude $\mathcal{A}_{B}$. With this resolution, we are interested in determining the amplitudes of the beam displacement $r(t)$ and the voltage $v(t)$. The experimental validation of the model is detailed in the next section.

\section{Experiments and results}

\subsection{Device introduction}

The experimental validation was carried out on the strongly coupled cantilever based on PMN-PT single crystals shown in Figure 3. The PMN-PT material we used is the [001] poled PMN-0.29PT produced by TRS ceramics (TRS X2B). The PMN-PT patches have been cut to size by the manufacturer and glued in our laboratory on a steel beam with epoxy glue (Epotecny E505). The proof mass is made from two steel sheets bonded on the substrates with a $3 \mathrm{M}^{\circledR}$ epoxy glue. The geometrical parameters are given in Table 1 and the material parameters are given in Table 2 .

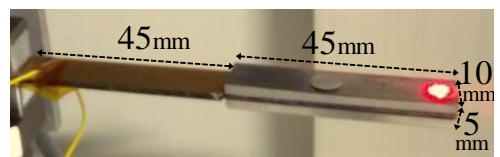

Figure 3 : Picture of the assembled prototype. 


\begin{tabular}{|c|c|}
\hline \multicolumn{2}{|c|}{$\begin{array}{l}\text { Table 1: Geometrical parameters of the } \\
\text { prototype. }\end{array}$} \\
\hline Material & PMN-PT \\
\hline Beam length $L_{b}$ & $45 \mathrm{~mm}$ \\
\hline Mass length $L_{m}$ & $45 \mathrm{~mm}$ \\
\hline Height of mass $H_{m}$ & $5 \mathrm{~mm}$ \\
\hline Beam and mass width $B$ & $10 \mathrm{~mm}$ \\
\hline Substrate thickness $h_{s}$ & $0.5 \mathrm{~mm}$ \\
\hline Piezoelectric thickness $h_{p}$ & $0.5 \mathrm{~mm}$ \\
\hline
\end{tabular}

\begin{tabular}{cc}
\hline Table 2: Properties of the PMN-PT \\
material [27]. \\
\hline$d_{3 I}$ & $-699{\mathrm{pm} . \mathrm{V}^{-1}}^{E}$ \\
$s_{11}^{E}$ & $52.1 \times 10^{-12} \mathrm{~Pa}^{-1}$ \\
$\epsilon_{33}^{T}$ & $5400 \epsilon_{0} \mathrm{~F}^{-1}$ \\
$\rho_{p}$ & $7750 \mathrm{~kg} \cdot \mathrm{m}^{-3}$ \\
\hline
\end{tabular}

Based on the material parameters given in Table 2, the expected values of the linear global parameters can be determined from equations (34) to (39) and are given in Table 3.

Table 3 : Coefficients deduced from the model and the material parameters.

\begin{tabular}{ccc}
\hline Name & Coefficient & Value \\
\hline Equivalent mass & $M$ & $35.4 \mathrm{~g}$ \\
Forcing term & $B_{f}$ & $25.4 \mathrm{~g}$ \\
Linear stiffness & $K$ & $1358 \mathrm{~N} / \mathrm{m}$ \\
Coupling term & $\Theta$ & $-3.70 \times 10^{-3} \mathrm{~N} / \mathrm{V}$ \\
Capacitance & $C_{p}$ & $69.1 \times 10^{-9} \mathrm{~F}$ \\
\hline
\end{tabular}

\subsection{Measurements under vibration and results}

The realized experiments consist in measuring the output power and the displacement amplitude under harmonic vibration excitation for various accelerations levels and various resistive loads. The experimental setup, depicted in Figure 4, is composed of an electromagnetic shaker (K2075E-HT), an accelerometer (PCB Piezotronics 356A17) are a programmable electrical resistance that are controlled from a computer thanks to a dSpace board. A dedicated Matlab script defines the acceleration level, frequency and control the resistive loads. The experiments have been done for 5 acceleration levels between $0.019 \mathrm{~m} / \mathrm{s}^{2}$ and $0.5 \mathrm{~m} / \mathrm{s}^{2}$ and 60 resistive loads $R_{\text {load }}$ logarithmically spaced between $1 \mathrm{k} \Omega$ and $10 \mathrm{M} \Omega$ over 95 excitation frequencies between $27 \mathrm{~Hz}$ and $33 \mathrm{~Hz}$.

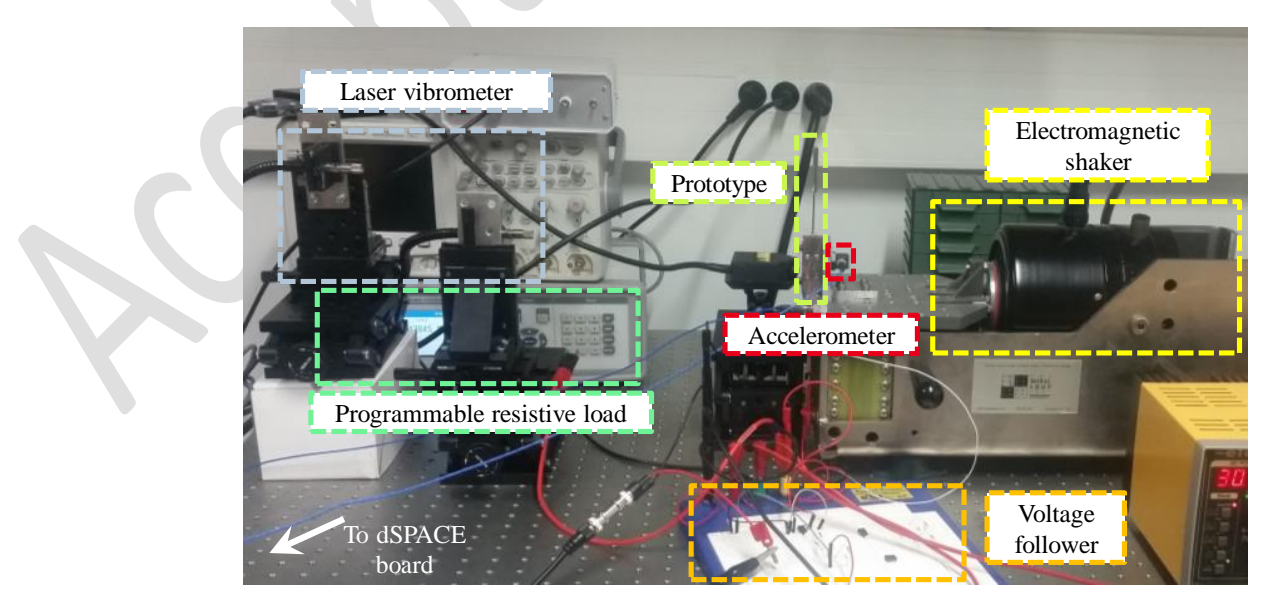

Figure 4 : Experimental setup for characterization under vibrations.

The harvested power is represented in Figure 5 as a function of the resistive load $R_{\text {load }}$ and the excitation frequency $f_{\text {ext }}$ for the 5 acceleration amplitudes $\mathcal{A}_{B}$. As the proposed prototype has a strong global electromechanical coupling coefficient $k^{2}$ and a high quality factor $Q_{m} \quad\left(\left[\frac{k^{2}}{1-k^{2}}\right] Q_{m}>9\right.$ as $k^{2}=16 \%$ and $Q_{m}>$ 50 ), two power peaks are observed. One power peak is reached near the short-circuit resonant frequency (about 
$29 \mathrm{~Hz}$ ) for a small resistive load (about $10 \mathrm{k} \Omega$ ), the second peak is reached near the open-circuit resonant frequency (about $31.5 \mathrm{~Hz}$ ) for a high resistive load (about $1.5 \mathrm{M} \Omega$ ). As the prototype exhibits mechanical softening, the power peaks occur at lower frequencies with increasing acceleration level.
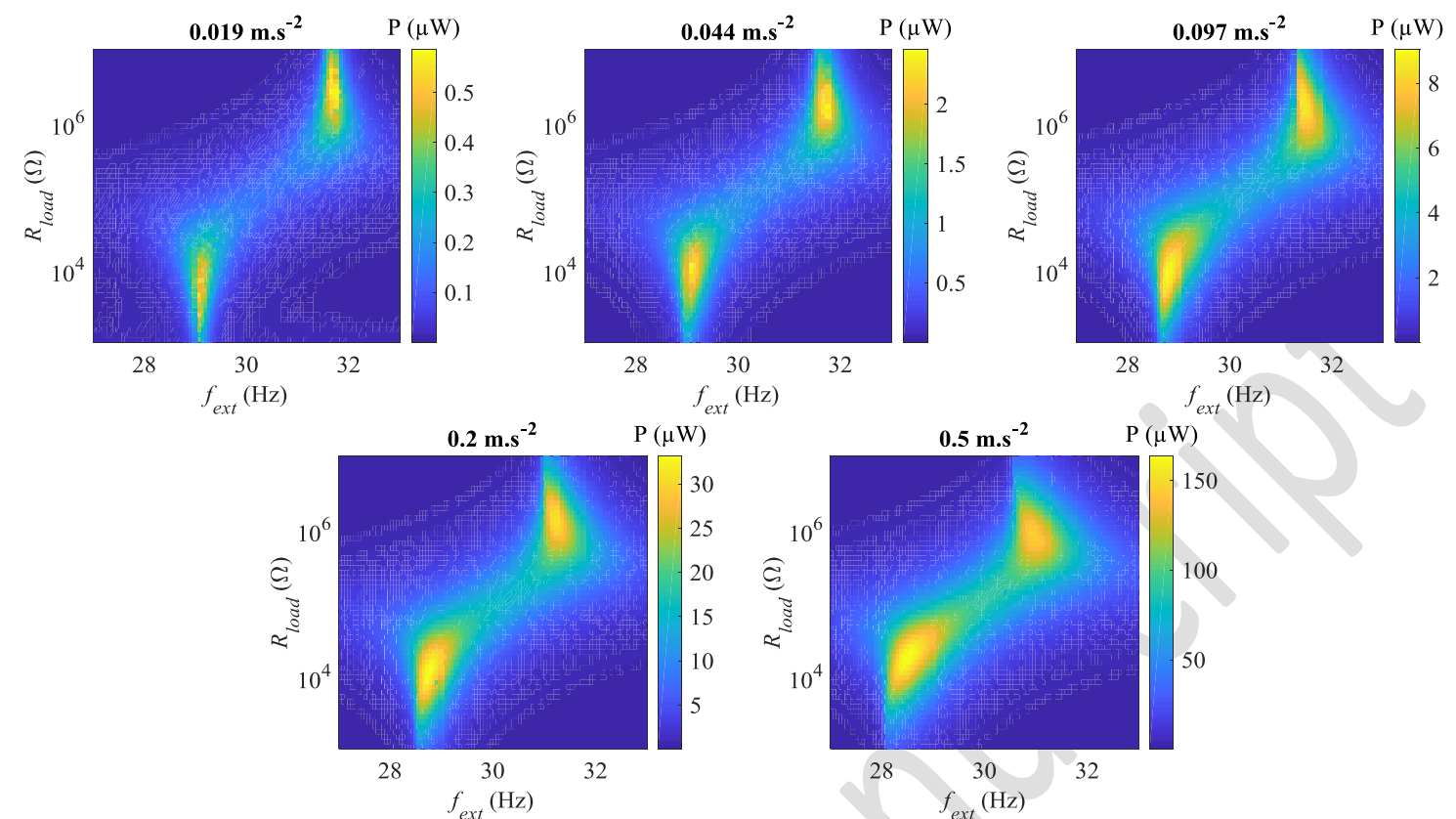

Figure 5 : Measured mean power as function of frequency and resistive load for 5 acceleration amplitudes $\mathcal{A}_{B}$. The mean power is given by $P=V^{2} / R_{\text {load }}$.

The experimental equivalent parameters of the equations (32) and (33) have been determined by fitting the power and displacement amplitude deduced from the model to the measured values. For this purpose, the amplitude error between the model mean power and the experiment was minimised by varying the equivalent parameters. The measured power and displacement for several resistive loads are represented in Figure 6 and Figure 7 respectively. The parameters derived from the fit are given in Table 4.
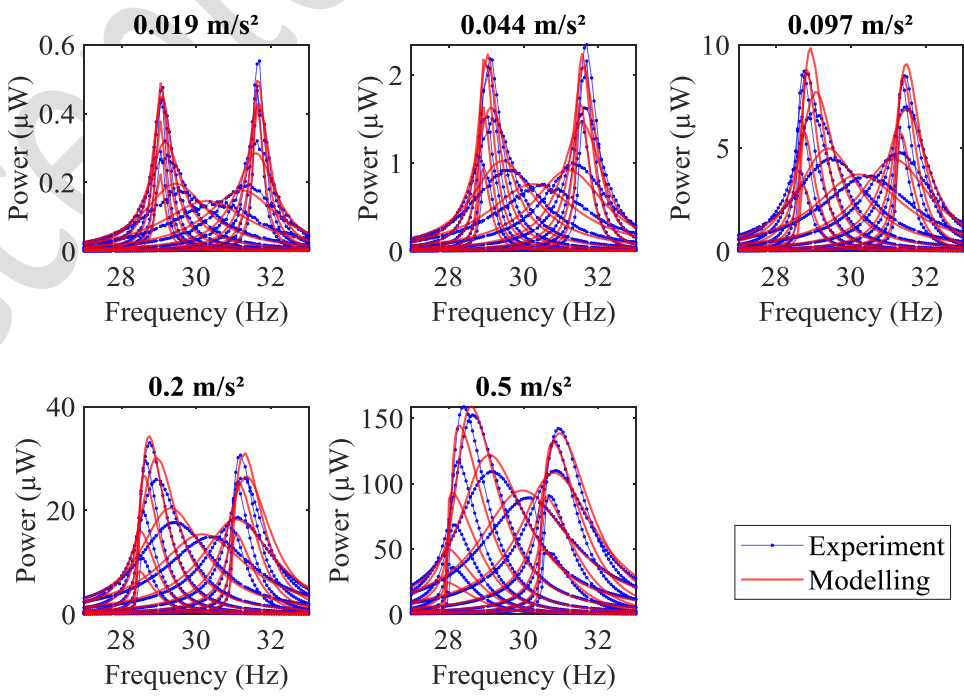

Figure 6 : Output mean power as a function of the excitation frequency for resistive loads of $1.2 \mathrm{k} \Omega, 2.6 \mathrm{k} \Omega$, $5.6 \mathrm{k} \Omega, 12 \mathrm{k} \Omega, 27 \mathrm{k} \Omega, 57 \mathrm{k} \Omega, 126 \mathrm{k} \Omega, 276 \mathrm{k} \Omega, 602 \mathrm{k} \Omega, 1.3 \mathrm{M} \Omega, 2.9 \mathrm{M} \Omega$ and $6.3 \mathrm{M} \Omega$ : experimental results and model results with fitted coefficients. 

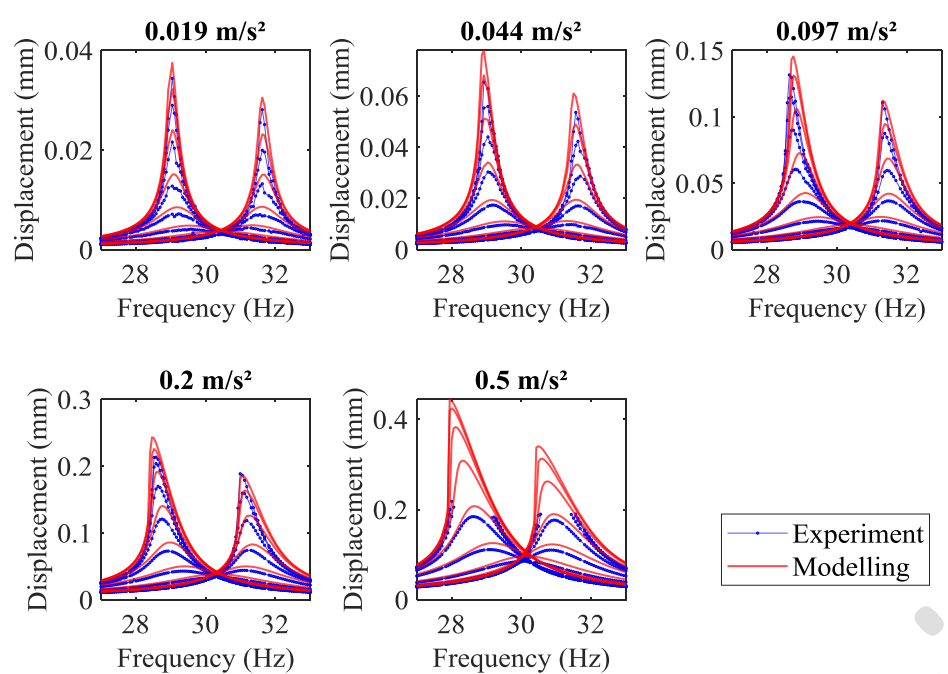

Figure 7 : Displacement amplitude $R$ as a function of the excitation frequency for resistive loads of $1.2 \mathrm{k} \Omega$, $2.6 \mathrm{k} \Omega, 5.6 \mathrm{k} \Omega, 12 \mathrm{k} \Omega, 27 \mathrm{k} \Omega, 57 \mathrm{k} \Omega, 126 \mathrm{k} \Omega, 276 \mathrm{k} \Omega, 602 \mathrm{k} \Omega, 1.3 \mathrm{M} \Omega, 2.9 \mathrm{M} \Omega$ and $6.3 \mathrm{M} \Omega$ : experimental results and model results with fitted coefficients. At $0.5 \mathrm{~m} / \mathrm{s}^{2}$, points are missing from the displacement measurement as the displacement could be only measured in the $\pm 0.2 \mathrm{~mm}$ range.

Table 4 : Coefficients deduced from the experiments.

\begin{tabular}{ccc}
\hline Name & Coefficient & Value \\
\hline Equivalent mass & $M$ & $35.2 \mathrm{~g}$ \\
Forcing term & $B_{f}$ & $23.1 \mathrm{~g}$ \\
Linear stiffness & $K$ & $1178 \mathrm{~N} / \mathrm{m}$ \\
Coupling term & $\Theta$ & $-3.05 \times 10^{-3} \mathrm{~N} / \mathrm{V}$ \\
Capacitance & $C_{p}$ & $41.9 \times 10^{-9} \mathrm{~F}$ \\
Nonlinear stiffness & $K_{2}$ & $-2.44 \times 10^{5} \mathrm{~N} / \mathrm{m}^{2}$ \\
Nonlinear coupling term & $\Theta_{2}$ & $1,22 \mathrm{~N} / \mathrm{Vm}$ \\
Linear mechanical loss & $b_{1}$ & $12.8 \mathrm{~N} / \mathrm{m}$ \\
Nonlinear mechanical loss & $b_{2}$ & $8,57 \times 10^{4} \mathrm{~N} / \mathrm{m}^{2}$ \\
Linear dielectric loss & $d_{1}$ & $0 \mathrm{~F}$ \\
Nonlinear dielectric loss & $d_{2}$ & $2,03 \times 10^{-10} \mathrm{~F} / \mathrm{V}$ \\
\hline
\end{tabular}

The average error between the model and experiment is calculated using equation (50) where $n_{\text {acc }}, n_{\text {res }}$ and $n_{f r q}$ are the numbers of accelerations, resistances and frequencies which are equal to 5, 60 and 95 respectively. $P_{\text {exp }}$ is the experimental mean power and $P_{t h}$ is the mean power derived from the model.

$$
\frac{1}{n_{a c c} n_{r e s} n_{f r q}} \sum^{n_{a c c}} \sum^{n_{r e s}} \sum^{n_{f r q}}\left(\frac{\left|P_{t h}-P_{\text {exp }}\right|}{P_{\text {exp }}}\right)
$$

The average error the model and experiment is equal to $9.5 \%$ for power evaluation. The average error for the displacement evaluation is equal to $14.4 \%$ for the 4 exploitable acceleration levels (i.e. measurements the $0.5 \mathrm{~m} / \mathrm{s}^{2}$ is not considered due to missing points).

\section{Discussions}

\subsection{Discussion on model validation}

It can be seen from Figure 6 and Figure 7 that the proposed model provides a good evaluation of the experimental behaviour of the cantilever-type generator. The presented model is therefore able to predict the nonlinear behaviour of strongly coupled harvesters subjected to various acceleration levels. 
Furthermore, the linear parameter deduced from model fitting (Table 3) are close to the linear parameters deduced from the material parameters of the material supplier (Table 4), which means that the model is also able to approximately predict the performance of a harvester in a design phase from the parameters given a priori. The differences, especially on the linear stiffness $K$ and linear coupling term $\Theta$ can be explained by uncertainties in material parameters, manufacturing and clamping conditions as discussed in previous work [12].

\subsection{Dielectric losses consideration}

It can be noted that the linear dielectric loss coefficient $d_{1}$ is taken as zero in the model fitting (Table 4). The nonlinear dielectric loss coefficient seems to be more relevant to consider the harvester behavior than the linear one. In order to analyze the influence of the nonlinear dielectric loss coefficient on the harvested power, the power at optimal resistive loads is represented in Figure 8 as a function of the frequency for 3 acceleration levels. The harvested power is shown with the consideration of the dielectric loss coefficient $d_{2}$ and without its consideration. Not taking the nonlinear dielectric loss coefficient into account is equivalent to using the model presented in [19].

The power for optimal pairs of parallel capacitive and resistive loads is also plotted to deduce the expected results when the resonant frequency of the harvester is electrically tuned. Indeed, non-linear electrical interfaces, such as those based on Synchronous Electric Charge Extraction (SECE) techniques, are able to emulate combinations of resistive and capacitive loads in order to tune the resonant frequency of strongly coupled piezoelectric harvesters [28]. The harvested power for resistive and capacitive loads is a relevant indicator that allows us to know the expected power using a non-linear electrical interface. As an example, the model predicts that the frequency bandwidths defined at half of the maximal power are equal to $3.1 \mathrm{~Hz}$ and $3.7 \mathrm{~Hz}$ for optimal resistive and capacitive loads and acceleration amplitudes $\mathcal{A}_{B}$ of $0.019 \mathrm{~m} / \mathrm{s}^{2}$ and $0.5 \mathrm{~m} / \mathrm{s}^{2}$ respectively (Figure 8). This represents relative bandwidths of $10 \%$ and $12.3 \%$ of the center frequencies respectively. This bandwidths corresponds to the minimum bandwidth expected when using the short-circuit synchronous electric charge extraction (SC-SECE) technique [9]. The expected behavior of a strongly coupled harvester with material nonlinearities has been validated experimentally with combinations of resistive and capacitive loads in a previous work [29].
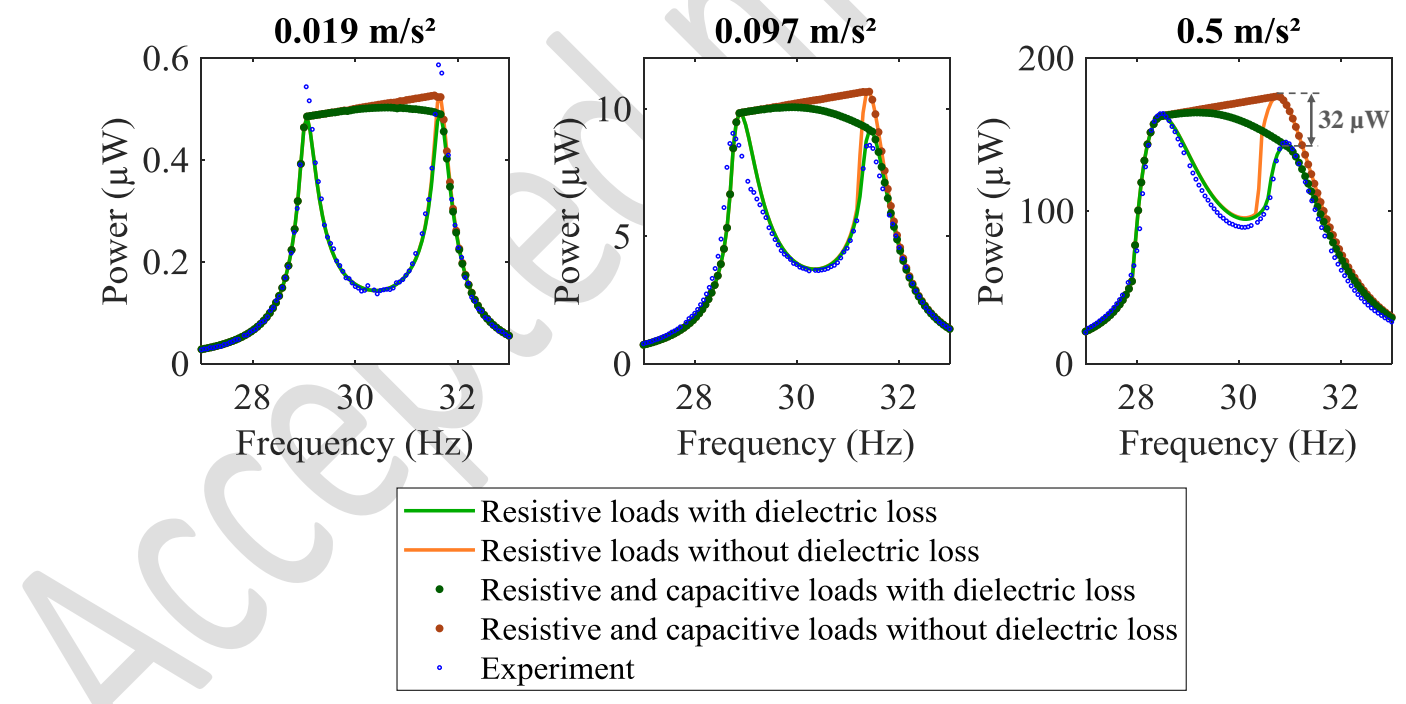

Figure 8: Model-derived power harvested by the proposed piezoelectric cantilever versus frequency for optimal resistive loads and optimal pairs of parallel capacitive and resistive loads.

The average errors between the models and experiment on power with optimal resistive loads are calculated for the three acceleration levels and are given in Table 5.

Table 5 : Average error between the models and experiment on power with optimal resistive loads

\begin{tabular}{cccc}
\hline Acceleration amplitude & $0.019 \mathrm{~m} / \mathrm{s}^{2}$ & $0.097 \mathrm{~m} / \mathrm{s}^{2}$ & $0.5 \mathrm{~m} / \mathrm{s}^{2}$ \\
\hline Average error of the model with dielectric losses & $3,6 \%$ & $7,1 \%$ & $6,5 \%$ \\
Average error of the model without dielectric losses & $3,4 \%$ & $8,6 \%$ & $12,3 \%$ \\
\hline
\end{tabular}


The proposed model offers a better accuracy on the power evaluation than the literature model with the increase of the acceleration amplitude. From the model (Figure 8), we can see that the nonlinear dielectric loss coefficient has a significant influence on the power harvested near the open-circuit resonant frequency (around $31.5 \mathrm{~Hz}$ ). This is because the load impedance magnitudes required to maximize the power are higher near the open-circuit resonant frequency and lower near to the short-circuit resonant frequency. This means that the optimal voltage close to $31.5 \mathrm{~Hz}$ is higher than the optimal voltage close to $29 \mathrm{~Hz}$, as shown in Figure 9. Therefore, since the energy loss per cycle increase with the voltage amplitude when we take into account dielectric losses, lower output power is expected around $31.5 \mathrm{~Hz}$ compared to the case when these losses are not taken into account.
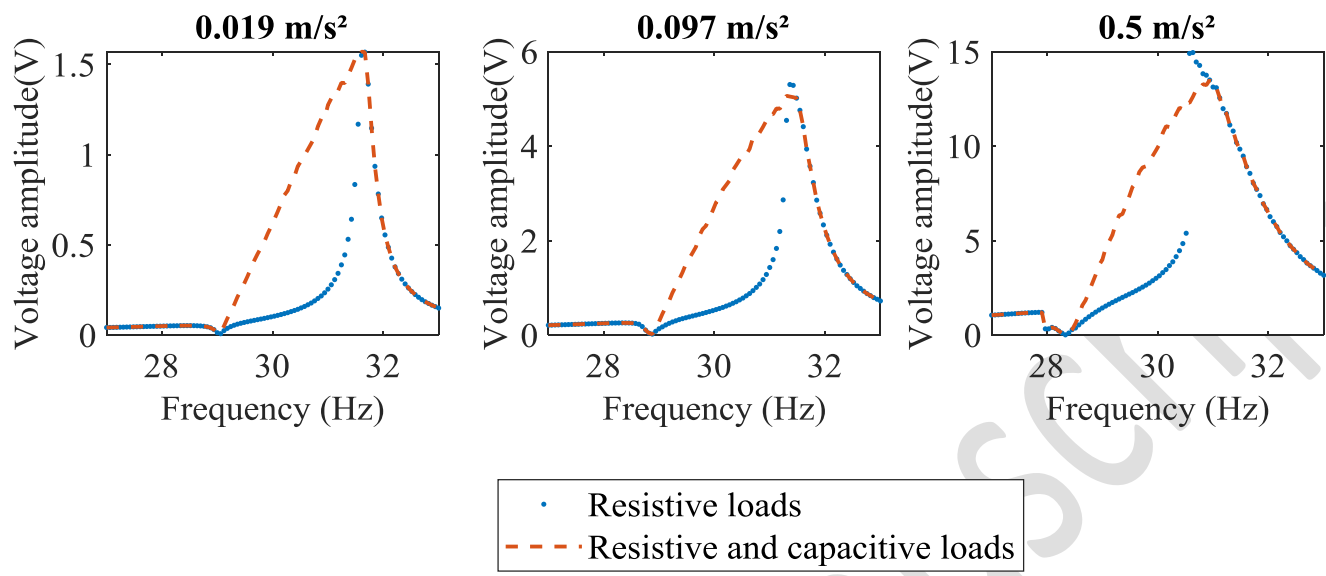

Figure 9 : Voltage magnitude derived from the model as a function of frequency for optimal resistive loads and optimal pairs of parallel capacitors and resistors.

The model (Figure 8) also shows that the influence of dielectric losses increases with the acceleration level. While the influence is hardly noticeable at low level $\left(0.019 \mathrm{~m} / \mathrm{s}^{2}\right)$, a strong influence is noticed at high acceleration level $\left(0.5 \mathrm{~m} / \mathrm{s}^{2}\right)$. Considering the dielectric losses, the maximum power harvested at the high optimal resistive load is $32 \mu \mathrm{W}$ lower than the maximum power that would be harvested without dielectric losses at the optimal reactive load at $0.5 \mathrm{~m} / \mathrm{s}^{2}$ (Figure 8). This represents a relative decrease of more than $18 \%$ of the expected power at the optimal resistive load. Neglecting nonlinear dielectric losses when modelling strongly coupled vibration energy harvesters can therefore lead to a significant overestimation of the harvested power.

Figure 10 represents the evolution of the calculated power at the second power peak on the optimal resistive load (the peaks around $31.5 \mathrm{~Hz}$ ) as a function of the acceleration level when the dielectric loss coefficient $d_{2}$ is taken into account and not taken into account. The relative decrease when considering the dielectric loss coefficient is also shown. This figure shows the increasing influence of the dielectric loss with the acceleration level. The relative power loss due to dielectric loss increases rapidly (15\% decrease over $\left.0.12 \mathrm{~m} / \mathrm{s}^{2}\right)$ and tends to saturate below $20 \%$ above $0.5 \mathrm{~m} / \mathrm{s}^{2}$. As this relative power decrease is not constant with the acceleration level variation, a linear dielectric loss coefficient cannot be sufficient to consider the increasing loss with voltage. Non-linear dielectric loss modelling is thus necessary to adequately account for the behavior of strongly coupled harvesters based on strongly coupled materials. As the behavior of strongly coupled harvester may differ depending on the piezoelectric material used, an analysis using this model on different types of materials should be carried out. 


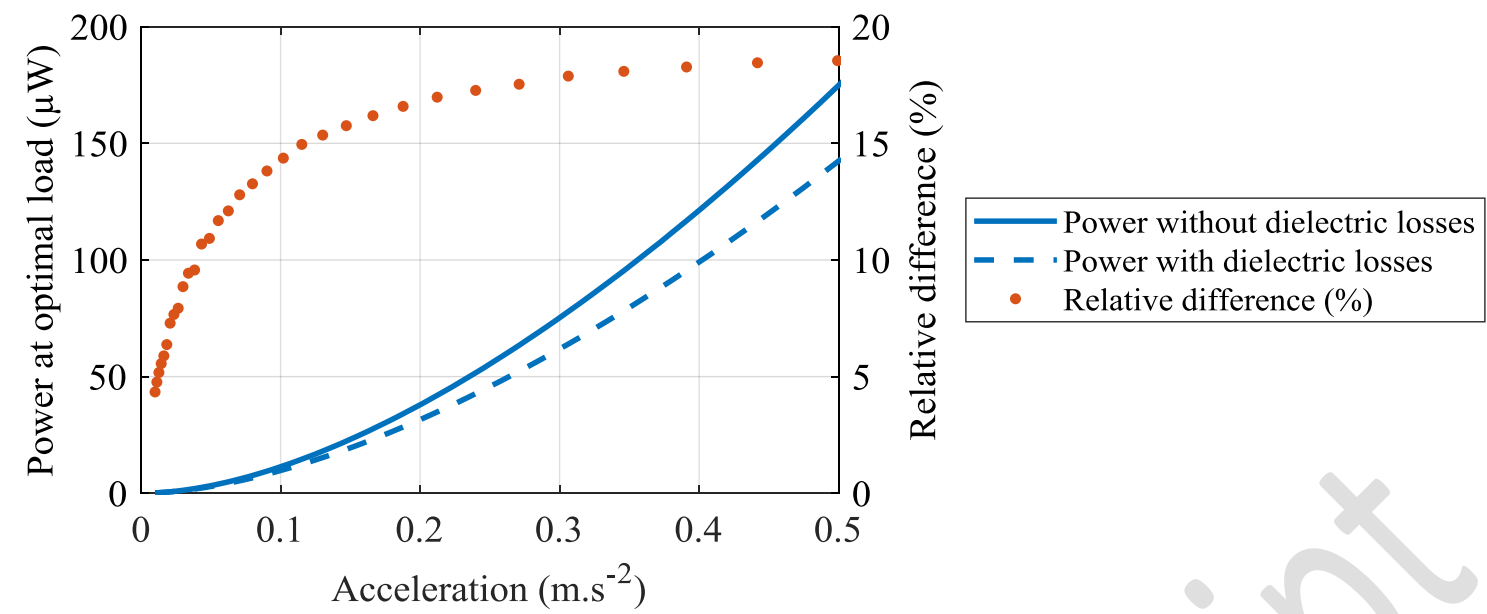

Figure 10 : Harvested power deduced from the model at the second power peak (around $31.5 \mathrm{~Hz}$ ) as a function of the acceleration amplitude $\mathcal{A}_{B}$. The relative difference is given by $\left|P_{\text {without }}-P_{\text {with }}\right| / P_{\text {with }}$ where $P_{\text {without }}$ is the harvested power without the consideration of dielectric losses and $\mathrm{P}_{\text {with }}$ is the harvested power with the consideration of dielectric losses

\section{Conclusions}

This paper reports on the experimental validation of a new model that considers nonlinear material losses in piezoelectric harvesters. For this purpose, it takes into account a second order dielectric loss term in addition to a mechanical second order mechanical loss term. The presented model allows a better consideration of the harvested power under vibration than previous models and is proven to be necessary for accurately predicting the performance of strongly coupled harvesters. An experimental validation is carried out with a strongly coupled cantilever based on PMN-PT material. The results reveal the importance of considering a nonlinear dielectric loss coefficient. Indeed, we show thanks to the proposed model that neglecting this coefficient can induce an error up to $18 \%$ between the measured power and the predicted power for the presented harvester.

As it has been shown that strongly coupled vibrational energy harvesters are needed to extend the performance of resonant frequency tuning by electrical methods, the use of the present model will be necessary for the study of broadband vibration harvesters. In future work, the effect of nonlinear dielectric loss on the power harvested by strongly coupled energy harvesters combined with advanced energy extraction circuit able to tune the generator's resonant frequency will be studied (e.g. SC-SECE).

\section{References}

[1] R. Torah, P. Glynne-Jones, M. Tudor, T. O’Donnell, S. Roy, S. Beeby, Self-powered autonomous wireless sensor node using vibration energy harvesting, Meas. Sci. Technol. 19 (2008) 125202. https://doi.org/10.1088/0957-0233/19/12/125202.

[2] G. Clementi, G. Lombardi, S. Margueron, M.A. Suarez, E. Lebrasseur, S. Ballandras, J. Imbaud, F. Lardet-Vieudrin, L. Gauthier-Manuel, B. Dulmet, M. Lallart, A. Bartasyte, LiNbO3 films - A low-cost alternative lead-free piezoelectric material for vibrational energy harvesters, Mech. Syst. Signal Process. 149 (2021) 107171. https://doi.org/10.1016/j.ymssp.2020.107171.

[3] G. Lombardi, M. Lallart, M. Kiziroglou, E.M. Yeatman, A piezoelectric self-powered active interface for AC/DC power conversion improvement of electromagnetic energy harvesting, Smart Mater. Struct. 29 (2020) 117002. https://doi.org/10.1088/1361-665X/abadd3.

[4] S.J. Roundy, Energy scavenging for wireless sensor nodes with a focus on vibration to electricity conversion, PhD Thesis, University of California, Berkeley Berkeley, CA, 2003.

[5] X. Li, D. Upadrashta, K. Yu, Y. Yang, Analytical modeling and validation of multi-mode piezoelectric energy harvester, Mech. Syst. Signal Process. 124 (2019) 613-631. https://doi.org/10.1016/j.ymssp.2019.02.003.

[6] L. Tang, Y. Yang, C.K. Soh, Toward Broadband Vibration-based Energy Harvesting, J. Intell. Mater. Syst. Struct. 21 (2010) 1867-1897. https://doi.org/10.1177/1045389X10390249. 
[7] T. Huguet, M. Lallart, A. Badel, Orbit jump in bistable energy harvesters through buckling level modification, Mech. Syst. Signal Process. 128 (2019) 202-215. https://doi.org/10.1016/j.ymssp.2019.03.051.

[8] A. Brenes, A. Morel, D. Gibus, C.-S. Yoo, P. Gasnier, E. Lefeuvre, A. Badel, Large-bandwidth piezoelectric energy harvesting with frequency-tuning synchronized electric charge extraction, Sens. Actuators Phys. 302 (2020) 111759. https://doi.org/10.1016/j.sna.2019.111759.

[9] A. Morel, G. Pillonnet, P. Gasnier, E. Lefeuvre, A. Badel, Frequency tuning of piezoelectric energy harvesters thanks to a short-circuit synchronous electric charge extraction, Smart Mater. Struct. 28 (2018) 025009. https://doi.org/10.1088/1361-665X/aaf0ea.

[10] Y. Cai, Y. Manoli, A piezoelectric energy-harvesting interface circuit with fully autonomous conjugate impedance matching, $156 \%$ extended bandwidth, and $0.38 \mu \mathrm{W}$ power consumption, in: 2018 IEEE Int. Solid - State Circuits Conf. - ISSCC, 2018: pp. 148-150. https://doi.org/10.1109/ISSCC.2018.8310227.

[11] A. Morel, A. Quelen, C.A. Berlitz, D. Gibus, P. Gasnier, A. Badel, G. Pillonnet, Fast-Convergence SelfAdjusting SECE Circuit With Tunable Short-Circuit Duration Exhibiting 368\% Bandwidth Improvement, IEEE Solid-State Circuits Lett. 3 (2020) 222-225. https://doi.org/10.1109/LSSC.2020.3012340.

[12] D. Gibus, P. Gasnier, A. Morel, F. Formosa, L. Charleux, S. Boisseau, G. Pillonnet, C.A. Berlitz, A. Quelen, A. Badel, Strongly coupled piezoelectric cantilevers for broadband vibration energy harvesting, Appl. Energy. 277 (2020) 115518. https://doi.org/10.1016/j.apenergy.2020.115518.

[13] Y. Kuang, Z.J. Chew, J. Dunville, J. Sibson, M. Zhu, Strongly coupled piezoelectric energy harvesters: Optimised design with over $100 \mathrm{~mW}$ power, high durability and robustness for self-powered condition monitoring, Energy Convers. Manag. 237 (2021) 114129. https://doi.org/10.1016/j.enconman.2021.114129.

[14] A. Morel, G. Pillonnet, Y. Wanderoild, A. Badel, Dielectric Losses Considerations for Piezoelectric Energy Harvesting, (2018). https://doi.org/info:doi/10.1166/jolpe.2018.1562.

[15] R. Holland, Representation of Dielectric, Elastic, and Piezoelectric Losses by Complex Coefficients, IEEE Trans. Sonics Ultrason. 14 (1967) 18-20. https://doi.org/10.1109/T-SU.1967.29405.

[16] O. Freychet, S. Boisseau, F. Frassati, A. Morel, P. Gasnier, D. Gibus, A. Badel, G. Despesse, Efficient optimal load and maximum output power determination for linear vibration energy harvesters with a twomeasurement characterization method, Smart Mater. Struct. 29 (2019) 015003. https://doi.org/10.1088/1361-665X/ab516f.

[17] K. Uchino, S. Hirose, Loss mechanisms in piezoelectrics: how to measure different losses separately, IEEE Trans. Ultrason. Ferroelectr. Freq. Control. 48 (2001) 307-321. https://doi.org/10.1109/58.896144.

[18] M. Wild, M. Bring, E. Halvorsen, L. Hoff, K. Hjelmervik, The challenge of distinguishing mechanical, electrical and piezoelectric losses, J. Acoust. Soc. Am. 144 (2018) 2128-2134. https://doi.org/10.1121/1.5057443.

[19] S. Leadenham, A. Erturk, Unified nonlinear electroelastic dynamics of a bimorph piezoelectric cantilever for energy harvesting, sensing, and actuation, Nonlinear Dyn. 79 (2015) 1727-1743. https://doi.org/10.1007/s11071-014-1770-x.

[20] P. Shivashankar, S.B. Kandagal, Characterization of elastic and electromechanical nonlinearities in piezoceramic plate actuators from vibrations of a piezoelectric-beam, Mech. Syst. Signal Process. 116 (2019) 624-640. https://doi.org/10.1016/j.ymssp.2018.06.063.

[21] A. Badel, E. Lefeuvre, Nonlinear Conditioning Circuits for Piezoelectric Energy Harvesters, in: E. Blokhina, A. El Aroudi, E. Alarcon, D. Galayko (Eds.), Nonlinearity Energy Harvest. Syst. MicroNanoscale Appl., Springer International Publishing, Cham, 2016: pp. 321-359. https://doi.org/10.1007/978-3-319-20355-3_10.

[22] The Institute of Electrical and Electronics Engineers, IEEE Standard on Piezoelectricity, ANSIIEEE Std 176-1987. (1988). https://doi.org/10.1109/IEEESTD.1988.79638.

[23] G. Liu, S. Zhang, W. Jiang, W. Cao, Losses in ferroelectric materials, Mater. Sci. Eng. R Rep. 89 (2015) 1-48. https://doi.org/10.1016/j.mser.2015.01.002.

[24] D. Damjanovic, Hysteresis in piezoelectric and ferroelectric materials, Academic Press, 2006.

[25] L.J. Gong, C.L. Pan, Q.S. Pan, Z.H. Feng, Theoretical analysis of dynamic property for piezoelectric cantilever triple-layer benders with large piezoelectric and electromechanical coupling coefficients, J. Adv. Dielectr. 06 (2016) 1650017. https://doi.org/10.1142/S2010135X1650017X.

[26] A. Preumont, Mechatronics: Dynamics of Electromechanical and Piezoelectric Systems, Springer Netherlands, 2006. https://www.springer.com/gp/book/9781402046957 (accessed September 9, 2020).

[27] TRS Technologies, Inc., Http://Www.Trstechnologies.Com/Resources. (n.d.). http://www.trstechnologies.com/Resources (accessed September 19, 2018).

[28] A. Morel, A. Badel, R. Grézaud, P. Gasnier, G. Despesse, G. Pillonnet, Resistive and reactive loads' influences on highly coupled piezoelectric generators for wideband vibrations energy harvesting, J. Intell. Mater. Syst. Struct. 30 (2019) 386-399. https://doi.org/10.1177/1045389X18810802. 
[29] D. GIBUS, P. GASNIER, A. MOREL, S. BOISSEAU, A. BADEL, Nonlinearities influences on performances of a strongly-coupled piezoelectric generator for broadband vibration energy harvesting, in: 2019 19th Int. Conf. Micro Nanotechnol. Power Gener. Energy Convers. Appl. PowerMEMS, 2019: pp. 1-5. https://doi.org/10.1109/PowerMEMS49317.2019.61547410359. 